\title{
Framework for the simulation of Wireless Power Transmission over Ultra Wide Band links in multipath propagation environments in the vicinity of the human body
}

\author{
Thomas Cuyckens ${ }^{1 *}$ and Hendrik Rogier ${ }^{1}$ \\ ${ }^{1}$ Department of information technology (INTEC), iMinds / Ghent University
}

\begin{abstract}
SUMMARY
In this contribution a modular framework for Ultra Wide Band Wireless Power Transmission in proximity of the human body is presented. The goal of this work is to simplify the study of wireless links by considering antenna models, including radiation characteristics when the antenna is deployed on the human body, and the power transferred over them in multipath, shadowed propagation environments, such as powering textile antenna systems completely integrated inside garments. To obtain this goal, antenna links are partitioned in black box models for all elementary building blocks, such as antenna transfer functions, propagation channels, active components and more, that can be recombined as one sees fit. The recombination allows for accurate simulation of intricate systems. For small examples, considerable speed and memory gains were observed (a factor 6.20 in time and 1.75 in memory), while for more elaborate examples it is even impossible to simulate a reference solution.
\end{abstract}

KEY WORDS: Wireless Power Transmission, Ultra Wide Band, multipath

\section{INTRODUCTION}

Within the broad field of Wireless Power Transmission (WPT) research, different (overlapping) subdivisions can be identified. A separation can be made into near-field and far-field WPT, based on transmitter-receiver distance. Being independent of transmitter-receiver distance, efficiency is a key specification and therefore a lot of research efforts focus on very efficient rectifier circuits [1] and rectennas [2]. In this manuscript we will focus on far-field WPT, the effect of the antennas and the propagation environment, in particular in a body-centric context.

Historically, research into far-field WPT boomed in the 60s and 70s with mile stones such as W. Brown remotely powering a model helicopter using radio waves [3] and space agencies investigating the feasibility of solar power harvesting in space and beaming it back to earth. After a cool down period, research in WPT is booming again with research into practical problems such as the remote powering of hard to reach sensor networks [4], of textile antenna systems completely integrated inside garments, cable-free desks, near-field wireless charging standards [5, 6] and (passive) Radio Frequency IDentification (RFID) tags [1].

To power remote sensor networks, a low power source (per MHz) is preferable in order not to disturb other transmission systems or to create high power density hot spots. Ultra Wide Band (UWB $)^{\dagger}$ systems provide such low power transmission spectral densities. In this respect, the American

${ }^{*}$ Correspondence to: Department of information technology (INTEC), iMinds / Ghent University, Sint-Pietersnieuwstraat 41, 9000 Gent, Belgium. E-mail: thomas.cuyckens@ugent.be

Contract/grant sponsor: Agency for Innovation by Science and Technology in Flanders (IWT)

$\dagger$ an UWB system has a bandwidth exceeding the lesser of $500 \mathrm{MHz}$ and $20 \%$ of its centre frequency 
Federal Communications Commission (FCC) has approved the unlicensed use in the range $3.1 \mathrm{GHz}-$ $10.3 \mathrm{GHz}$. However, since such a large band nullifies the frequency independence assumption, common in small band antenna design, any description of UWB systems will have to inherently include frequency dependence and dispersion effects.

Research into far-field WPT is hampered by the fact that full-wave electromagnetic solvers are unable to simulate large and complex propagation environments within a reasonable amount of time, due to the discretization requirements of such problems. Therefore, we developed a new tool to calculate the WPT of an antenna link in a multipath environment in the vicinity of the human body, in contrast to previous contributions that mainly concentrate on UWB communication. Compared to existing link simulation tools as presented e.g. in [7, 8], we explicitly focus on the non-negligible frequency dependence of UWB channels and the power transferred over them. Previously, statistical approaches to UWB channels have been used [9, 10, 11], but they do not enable the designer to quantify the exact amount of power harvested for a given WPT system operating in well-known deterministic conditions, nor was shadowing of the human body taken into account. In [12, 13, 14] extensive research on body-centric communications has been performed. In [13], it is mentioned that link simulation tools are necessary to improve the design of communication applications in the vicinity of the body. Our contribution, centred on power transmission, helps to fill this gap for onbody links. Therefore, we present a modular framework in which frequency dependence (as required for UWB) and complex multipath behaviour (being the indoor environment and the proximity of the human body to the receive antenna) are unified in order to simulate and accurately predict WPT in a body-centric environment at a low computational cost.

We build, amongst others, on the work of [15, 16] to propose a framework that constructs frequencydependent antenna links based on stand-alone blocks in the vicinity of the human body. Each of the blocks can be a mathematical description, a simulation, a measurement or a third party black box description of an antenna together with antenna platform effects, such as the human body on which the antenna is deployed, a propagation channel (e.g. the statistical approaches to UWB propagation as mentioned before, or [17, 18]) or any building blocks forming part of the antenna link. This general approach allows developers to pick the best or most appropriate technique for each part and insert it into the model, but it also enables manufacturers to provide a component description without compromising intellectual property by disclosing a particular design.

We apply this approach to combine multipath channel models, as derived in Section 2 , with antenna simulations and antenna measurements on the human body to calculate the power transmission in the vicinity of the human body. We first verify our theory (Section 3 with a combination of measurements and simulations performed with Computer Simulation Technology's Microwave Studio ${ }^{\circledR}$ (CST-MWS ${ }^{\circledR}$ ) (CST-Computer Simulation Technology AG, Darmstadt, Germany). Then, we use the proposed framework to calculate the optimal position of a receiver placed on the human body to maximize the power transfer in a line-of-sight (LOS) and a non-line-of-sight (NLOS) propagation environment (Section 4)(Table \. More specifically we compare and interpret set-ups without, with one and with an abundance of reflections.

\section{THEORY}

In Fig. 1, a typical WPT link from generator to harvester (and storage) is depicted. It includes a generator, a transmitter $(\mathrm{Tx})$, a propagation channel $(\mathrm{CH})$, a receiver $(\mathrm{Rx})$, a matching network, a rectifier, a boost converter and a harvester with energy storage. Of interest here is the amount of power that can be harvested and stored given a certain amount of generated power. In this paper, we will analyse and optimize only part of this link, being the antenna link with some propagation channel, as enclosed by the dashed box in Fig. 11. Power loss in the matching network, rectifier or any other component outside the wireless link fall outside the scope of this research. The antenna link, presented in more detail in Fig. 2, is assumed to be terminated by a load impedance $Z_{L}\left(\Re Z_{L}=R_{L}\right)$. 


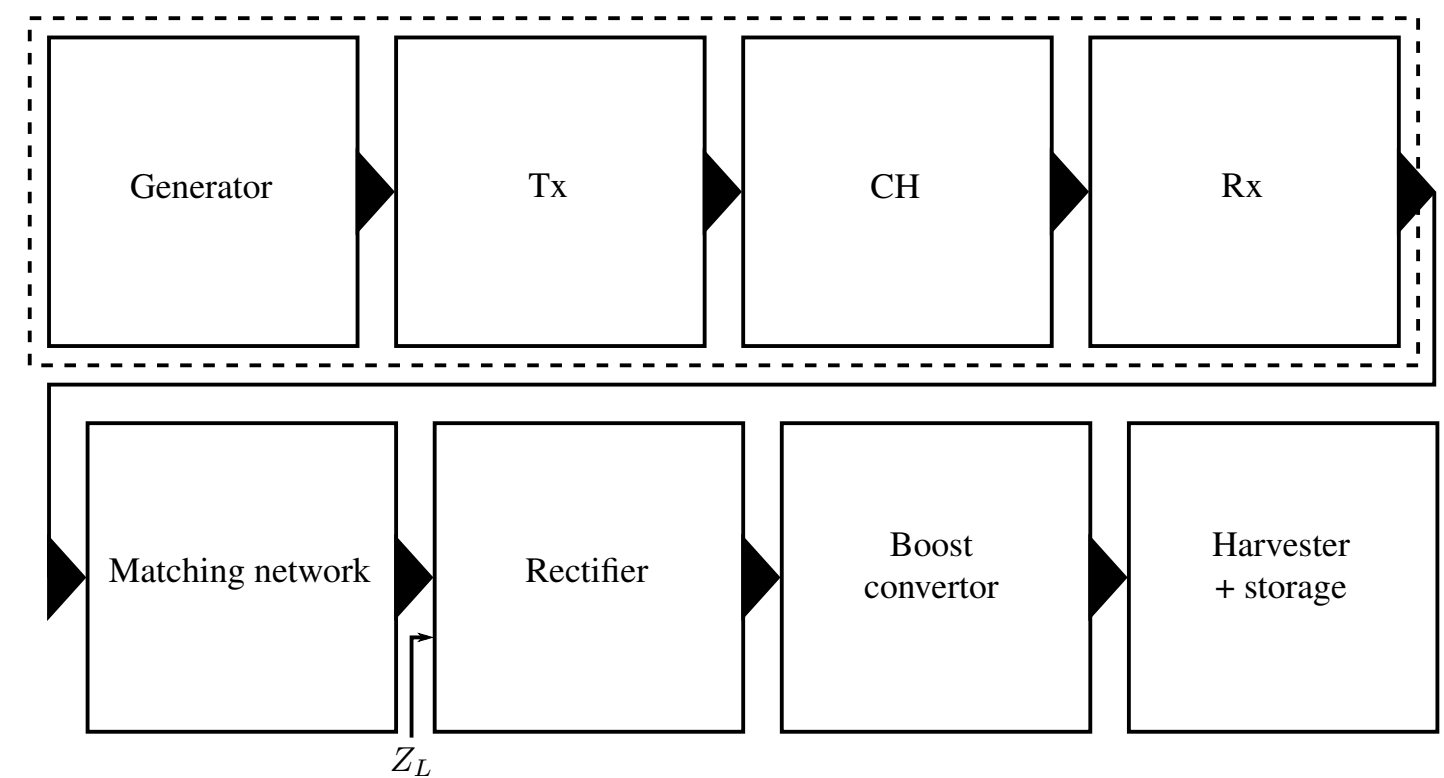

Figure 1. A WPT link from generator to harvester (and storage), including a generator, a transmit antenna $(\mathrm{Tx})$, a propagation channel $(\mathrm{CH})$, a receive antenna $(\mathrm{Rx})$, a matching network, a rectifier, a boost converter and a harvester with energy storage. This paper focusses on the optimization of the power transfer over the wireless link only (enclosed by the dashed box)

We will particularly focus on the ratio between the power harvested at the load $\left(P_{L}\right)$

$$
P_{L}=\frac{R_{L}}{2} \frac{\left|V_{o}\right|^{2}}{\left|Z_{L}\right|^{2}},
$$

where $V_{o}$ is the open circuit voltage of the receive antenna, further defined by (6) and the maximum available power $\left(P_{s}\right)$

$$
P_{s}=\frac{1}{8} \frac{\left|V_{s}\right|^{2}}{R_{s}}
$$

that may be delivered by the source $V_{s}$ (with impedance $Z_{s}$ and $\Re Z_{s}=R_{s}$ ) to a transmit antenna in ideal matching conditions. In reality, the transmit antenna has an internal impedance $Z_{T}$ and one might want to consider the actual power generated by $V_{s}$ or the power radiated by Tx.

When the transmitter and the receiver are both free standing antennas located in free space with only a direct path between them, the received power as a function of the maximum power delivered by the source's generator is described by the Friis transmission formula. In case of a UWB context, all quantities involved become frequency dependent, resulting in

$$
P_{R}(f)=M_{R}(f) \cdot G_{R}(f) \cdot Q_{R T}(f) \cdot\left(\frac{c}{4 \pi f d}\right)^{2} \cdot G_{T}(f) \cdot M_{T}(f) \cdot P_{T}(f),
$$

with $M_{R}, M_{T}, G_{R}$ and $G_{T}$ the mismatch factor $(M)$ and the power gain $(G)$ of the receive $\left.{ }_{R}\right)$ and the transmit ${ }_{T}$ ) antennas, respectively, $(4 \pi d f / c)^{2}$ the free space path loss, which depends on the distance $d$ between transmitter and receiver, $c$ the speed of light and $Q_{R T}$, the polarisation mismatch, defined as

$$
Q_{R T}=\frac{\left|\boldsymbol{F}_{R}(-\boldsymbol{u}) \circ \boldsymbol{F}_{T}(\boldsymbol{u})\right|^{2}}{\left|\boldsymbol{F}_{R}(-\boldsymbol{u})\right|^{2}\left|\boldsymbol{F}_{T}(\boldsymbol{u})\right|^{2}},
$$

with $\boldsymbol{F}_{R}(-\boldsymbol{u})$ and $\boldsymbol{F}_{T}(\boldsymbol{u})$ the radiation vector of the receive and transmit antenna, respectively, for a unit current and 'o' the scalar product. The radiation vector $\boldsymbol{F}(\boldsymbol{u})=\left[F^{V}(\boldsymbol{u}), F^{H}(\boldsymbol{u})\right]$ consists of a vertical $\left({ }^{V}\right)$ and horizontal $\left({ }^{H}\right)$ polarisation component. 


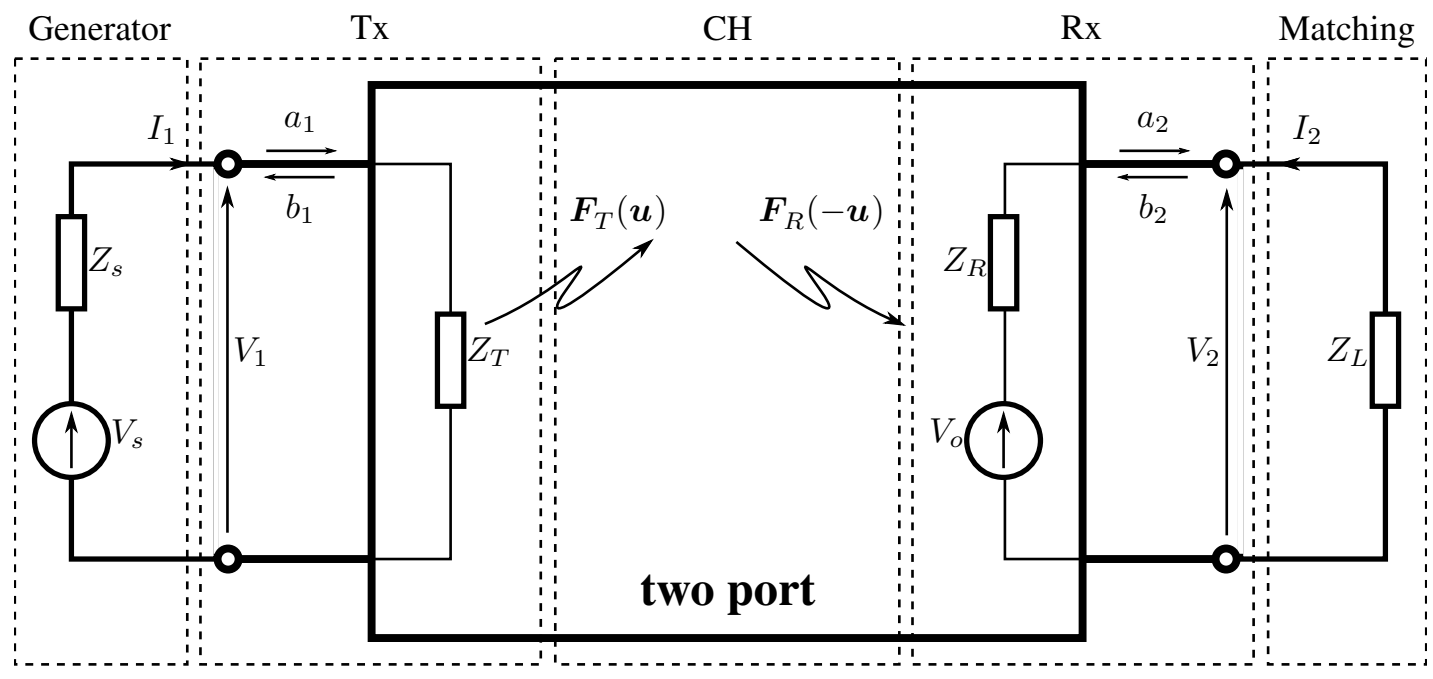

Figure 2. Schematics of a two-port network representation of the antenna link. The antenna link consists of a transmit antenna Tx (internal impedance $Z_{T}$ and far-field vector $\left.\boldsymbol{F}_{T}(\boldsymbol{u})\right)$ powered by a generator $\left(V_{s}\right.$ with impedance $Z_{s}$ ) and a receive antenna $\mathrm{Rx}$ (internal impedance $Z_{R}$ and far-field vector $\boldsymbol{F}_{R}(-\boldsymbol{u})$ ) terminated by a load $Z_{L}$.

The Friis transmission formula expresses power transmission in an ideal situation and is insufficient to express power transmission in a multipath environment or with antennas placed on the human body, located on or in walls or, in general, in 'complex' environments. In order to describe such a more intricate configuration, one has to derive the power transmission based on the voltages and currents on both antennas.

From Fig. 2, one can derive the voltage dissipated in the load $\left(Z_{L}\right)$ as

$$
V_{2}=\frac{Z_{L}}{Z_{L}+Z_{R}} V_{o}
$$

with $Z_{R}$ the internal impedance of the receiving antenna. The open circuit voltage $V_{o}$ is given by

$$
V_{o}=\frac{-2 \jmath c}{\eta f}\left(\boldsymbol{F}_{R}(-\boldsymbol{u}) \circ \boldsymbol{E}^{i n c}\right)
$$

with $\jmath$ the complex unit, $\eta$ the free space impedance, $\boldsymbol{E}^{i n c}$ the electric field of an impinging plane wave. From this point on, all radiation vectors will include the effect of all objects in the reactive near-field of the antenna, such as shadowing by the human body, but the notation for the radiation vector remains unaltered. Under the far-field assumption and in a multipath environment, $\boldsymbol{E}^{i n c}$ can be replaced by the electric field generated by the transmit antenna. This means

$$
\left(\boldsymbol{F}_{R}(-\boldsymbol{u}) \circ \boldsymbol{E}^{i n c}\right)=I_{1} \sum_{i} \frac{\mathrm{e}^{-\jmath k d_{i}}}{d_{i}}\left(\boldsymbol{F}_{R}\left(-\boldsymbol{u}_{i}\right) \circ \overline{\overline{\boldsymbol{\Gamma}}}_{i} \circ \boldsymbol{F}_{T}\left(\boldsymbol{u}_{i}\right)\right)
$$

with $k=2 \pi f / c$ the free space wave number, $\boldsymbol{u}_{i}$ and $-\boldsymbol{u}_{i}$ the transmit and receive direction of the $i^{\text {th }}$ path, respectively, $d_{i}$ the length of the $i^{\text {th }}$ path and $\overline{\overline{\boldsymbol{\Gamma}}}_{i}$ a full tensor containing the product of the $\gamma$ (numbered 0 to $\gamma-1$ ) reflections and transmissions along the $i^{\text {th }}$ path.

$$
\overline{\overline{\boldsymbol{\Gamma}}}_{i}=\prod_{j=0}^{\gamma-1} \begin{cases}\overline{\overline{\boldsymbol{R}}}_{i, \gamma-1-j} & \text { if the } j^{\text {th }} \text { interaction is a reflection } \\ \overline{\overline{\boldsymbol{T}}}_{i, \gamma-1-j} & \text { if the } j^{\text {th }} \text { interaction is a transmission }\end{cases}
$$

The index $j$ follows the $i^{\text {th }}$ path from transmitter to receiver as it goes through a concatenation of transmissions and reflections. This means, that if the $i^{\text {th }}$ path is first reflected on a surface and then twice transmitted through others, we have that $\overline{\overline{\boldsymbol{\Gamma}}}_{i}=\overline{\overline{\boldsymbol{T}}}_{i, 0} \overline{\overline{\boldsymbol{T}}}_{i, 1} \overline{\overline{\boldsymbol{R}}}_{i, 2}$. 
$\overline{\overline{\boldsymbol{R}}}_{i, j}$ and $\overline{\overline{\boldsymbol{T}}}_{i, j}$ are expressed in the same 'vertical-horizontal' base as the antenna polarisation. They are obtained by rotating the diagonal tensors $\overline{\boldsymbol{R}}_{i, j}^{D}=\operatorname{diag}\left(R_{i, j}^{\mathrm{TE}}, R_{i, j}^{\mathrm{TM}}\right)$ and $\overline{\overline{\boldsymbol{T}}}_{i, j}^{D}=\operatorname{diag}\left(T_{i, j}^{\mathrm{TE}}, T_{i, j}^{\mathrm{TM}}\right)$. The components of $\overline{\boldsymbol{R}}_{i, j}^{D}$ and $\overline{\overline{\boldsymbol{T}}}_{i, j}^{D}$ are the reflection and transmission coefficients of a wave with a polarisation parallel and perpendicular to the normal of the reflection plane. The reflection coefficients are given by

$$
\begin{aligned}
R_{i, j}^{\mathrm{TM}} & =\frac{\varepsilon_{r} \sin \psi_{i, j}-\sqrt{\varepsilon_{r}-\cos ^{2} \psi_{i, j}}}{\varepsilon_{r} \sin \psi_{i, j}-\sqrt{\varepsilon_{r}-\cos ^{2} \psi_{i, j}}} \\
R_{i, j}^{\mathrm{TE}} & =\frac{\sin \psi_{i, j}-\sqrt{\varepsilon_{r}-\cos ^{2} \psi_{i, j}}}{\sin \psi_{i, j}-\sqrt{\varepsilon_{r}-\cos ^{2} \psi_{i, j}}},
\end{aligned}
$$

with $\psi_{i, j}$ the angle of incidence of $i^{\text {th }}$ path at the $j^{\text {th }}$ transmission or reflection (see Fig. 5 ) and $\overline{\overline{\boldsymbol{T}}}_{i, j}^{D}=\overline{\overline{\mathbf{1}}}+\overline{\overline{\boldsymbol{R}}}_{i, j}^{D}$, with $\overline{\overline{\mathbf{1}}}$ the unity tensor. Note that the expression for $\overline{\overline{\boldsymbol{\Gamma}}}_{i}$ will be frequency dependent.

Expressing $I_{1}$ in $(7)$ as a function of the generator voltage $V_{s}$, the generator impedance $Z_{s}$ and the internal impedance of the transmit antenna $Z_{T}$ results in

$$
V_{2}=\frac{Z_{L}}{Z_{L}+Z_{R}} \frac{-2 \jmath c}{\eta f}\left[\sum_{i} \frac{\mathrm{e}^{-\jmath k d_{i}}}{d_{i}}\left(\boldsymbol{F}_{R}\left(-\boldsymbol{u}_{i}\right) \circ \overline{\overline{\boldsymbol{\Gamma}}}_{i} \circ \boldsymbol{F}_{T}\left(\boldsymbol{u}_{i}\right)\right)\right] \frac{1}{Z_{T}+Z_{s}} V_{s} .
$$

The polarisation mismatch can be written as $q$, the cosine of the angle between the radiation vectors of the transmitting and the receiving antenna, as given below

$$
q=\frac{\boldsymbol{F}_{T}\left(\boldsymbol{u}_{0}\right) \circ \boldsymbol{F}_{R}\left(-\boldsymbol{u}_{0}\right)}{\left|\boldsymbol{F}_{T}\left(\boldsymbol{u}_{0}\right)\right|\left|\boldsymbol{F}_{R}\left(-\boldsymbol{u}_{0}\right)\right|}
$$

with $\boldsymbol{F}_{R}\left(-\boldsymbol{u}_{0}\right)$ and $\boldsymbol{F}_{T}\left(\boldsymbol{u}_{0}\right)$ associated to the direct path and $Q=|q|^{2}$.

In order to bring the radiation vectors outside of the sum, one can replace $\overline{\overline{\Gamma_{i}}}$ by

$$
\Gamma_{i}^{\prime}=\frac{\boldsymbol{F}_{R}\left(-\boldsymbol{u}_{i}\right) \circ \overline{\overline{\boldsymbol{\Gamma}}}_{i} \circ \boldsymbol{F}_{T}\left(\boldsymbol{u}_{i}\right)}{\boldsymbol{F}_{R}\left(-\boldsymbol{u}_{0}\right) \circ \boldsymbol{F}_{T}\left(\boldsymbol{u}_{0}\right)},
$$

and thus

$$
\boldsymbol{F}_{R}\left(-\boldsymbol{u}_{i}\right) \circ \overline{\overline{\boldsymbol{\Gamma}}}_{i} \circ \boldsymbol{F}_{T}\left(\boldsymbol{u}_{i}\right)=\left|\boldsymbol{F}_{T}\left(\boldsymbol{u}_{0}\right)\right| \cdot q \cdot \Gamma_{i}^{\prime} \cdot\left|\boldsymbol{F}_{R}\left(-\boldsymbol{u}_{0}\right)\right|
$$

Then define $H_{R}$ and $H_{T}$ as below

$$
\begin{aligned}
\frac{V_{2}}{V_{s}} & =\underbrace{\left(\frac{\left|\boldsymbol{F}_{R}\left(-\boldsymbol{u}_{0}\right)\right|}{Z_{L}+Z_{R}}\right)}_{\left|H_{R}\right|} \underbrace{\left(\frac{-2 \jmath c}{\eta f} Z_{L} q \sum_{i} \Gamma_{i}^{\prime} \frac{\mathrm{e}^{-\jmath k d_{i}}}{d_{i}}\right)}_{H_{C H}} \underbrace{\left(\frac{\left|\boldsymbol{F}_{T}\left(\boldsymbol{u}_{0}\right)\right|}{Z_{T}+Z_{s}}\right)}_{\left|H_{T}\right|} .
\end{aligned}
$$

(15) is written down as a product of transfer functions, which mimics well the block model that was presented in Fig. 1. This property will be exploited later on.

Based on (15), (1) and (2), the ratio $P_{L}$ to $P_{s}$ is given by

$$
\frac{P_{L}}{P_{s}}=4 R_{L}\left|\frac{\boldsymbol{F}_{R}\left(-\boldsymbol{u}_{0}\right)}{Z_{L}+Z_{R}}\right|^{2}\left|\frac{-2 \jmath c}{\eta f} Z_{L} q \sum_{i} \Gamma_{i}^{\prime} \frac{\mathrm{e}^{-\jmath k d_{i}}}{d_{i}}\right|^{2}\left|\frac{\boldsymbol{F}_{T}\left(\boldsymbol{u}_{0}\right)}{Z_{s}+Z_{T}}\right|^{2} R_{s} .
$$

To use the theory above, one has to obtain data for the transfer function of an antenna. This means that for an antenna placed on the human body, the transfer function of the antenna on the body (instead of 'just the transfer function of the stand-alone antenna') should be measured or simulated. 
When relying on simulations, one uses the expression

$$
\boldsymbol{H}_{x}=\frac{\boldsymbol{F}_{x}(\boldsymbol{u})}{Z_{x}+Z_{L}}
$$

for antenna $x$ with internal impedance $Z_{x}$ and terminated by $Z_{L}$. To measure the transfer function of an antenna, a full 3D radiation vector of the antenna is needed. This quantity is obtained through a three-antenna measurement. Note that the three-antenna measurement has to be performed for the vertical and the horizontal polarisation. The three-antenna measurement consists of measuring the $S_{21}$ between any pair of the three antennas. From two-port theory, one can easily derive an expression for $S_{21}$ as a function of port voltages. Given the two-port in Fig. 2, the standard expressions for the $S$-parameters are given by 18

$$
S_{11}=\left.\frac{b_{1}}{a_{1}}\right|_{a_{2}=0} \quad S_{12}=\left.\frac{b_{1}}{a_{2}}\right|_{a_{1}=0} \quad S_{21}=\left.\frac{b_{2}}{a_{1}}\right|_{a_{2}=0} \quad S_{22}=\left.\frac{b_{2}}{a_{2}}\right|_{a_{1}=0} .
$$

and the necessary expressions for the forward and backwards normalized voltage waves given by (19) (with '*' the complex conjugate, $V_{s}=V_{1}+Z_{s} I_{1}, V_{2}=-Z_{L}^{*} I_{2}$ and under the assumption that the two-port is terminated by two impedances satisfying $\left.\left|\Re Z_{L}\right|=\left|\Re Z_{s}\right|\right)$.

$$
\begin{aligned}
a_{1} & =\frac{1}{2} \frac{V_{1}+Z_{s} I_{1}}{\sqrt{\left|\Re Z_{s}\right|}} & b_{1} & =\frac{1}{2} \frac{V_{1}-Z_{s}^{*} I_{1}}{\sqrt{\left|\Re Z_{s}\right|}} \\
a_{2} & =\frac{1}{2} \frac{V_{2}+Z_{L} I_{2}}{\sqrt{\left|\Re Z_{L}\right|}} & b_{2} & =\frac{1}{2} \frac{V_{2}-Z_{L}^{*} I_{2}}{\sqrt{\left|\Re Z_{L}\right|}}
\end{aligned}
$$

$S_{21}$ then yields

$$
S_{21}=\frac{b_{2}}{a_{1}}=\frac{\sqrt{\left|\Re Z_{s}\right|}\left(V_{2}-Z_{L}^{*} I_{2}\right)}{\sqrt{\left|\Re Z_{L}\right|}\left(V_{1}+Z_{s} I_{1}\right)}=\frac{2 V_{2}}{V_{s}} .
$$

Leveraging the block model, one can express $S_{21}$ for the vertical polarisation (the expression for the horizontal polarisation is similar) as

$$
S_{21}^{V}=\frac{2 V_{2}}{V_{s}}=2 H_{R}^{V} \cdot H_{C H}^{V} \cdot H_{T}^{V}
$$

Given three antennas, labelled $x, y$ and $z$, one can write down the relation above for every pair of antennas, resulting in a set of three equations (the notation for the polarisation is suppressed)

$$
\left\{\begin{array}{l}
S_{21}^{x y}=2 \cdot H_{x} \cdot H_{C H}^{x y} \cdot H_{y} \\
S_{21}^{y z}=2 \cdot H_{y} \cdot H_{C H}^{y z} \cdot H_{z} \\
S_{21}^{z x}=2 \cdot H_{z} \cdot H_{C H}^{z x} \cdot H_{x}
\end{array}\right.
$$

This can be solved for the different transfer functions. $H_{x}$ is given by

$$
H_{x}=\sqrt{\frac{1}{2} \frac{S_{21}^{x y}}{H_{C H}^{x y}} \frac{H_{C H}^{y z}}{S_{21}^{y z}} \frac{S_{21}^{z x}}{H_{C H}^{z x}}} .
$$

The expressions for $H_{y}$ and $H_{z}$ are the same, a permutation of antenna indices aside. Calculating the previous equation for both the vertical and the horizontal polarisation results in

$$
\boldsymbol{H}_{x}=\left[H_{x}^{V}, H_{x}^{H}\right] .
$$




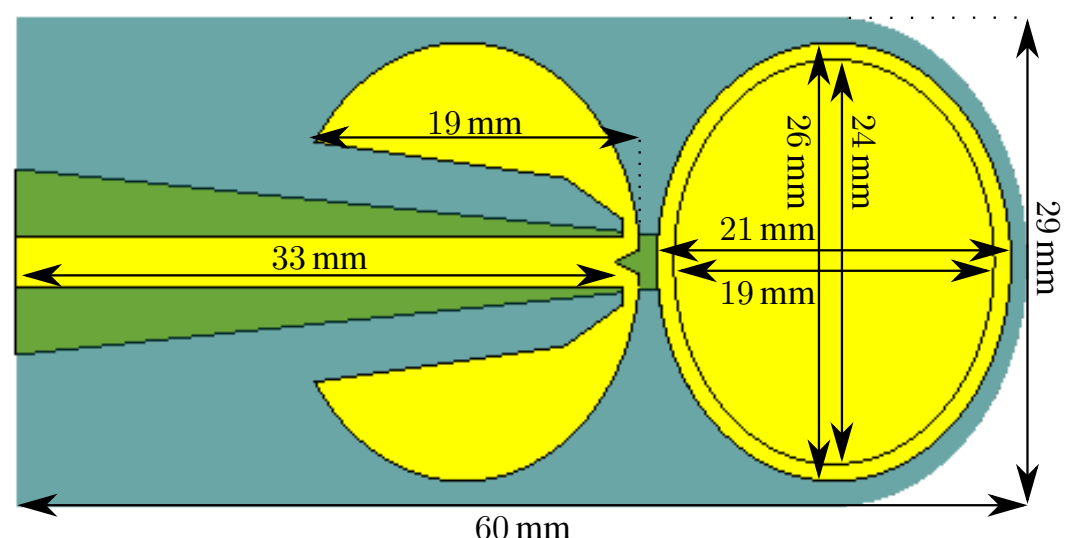

Figure 3. UWB monopole as recreated in CST-MWS ${ }^{\circledR}$

\section{THEORY VALIDATION}

As mentioned before, an antenna link can be described with a block model. In terms of power, the model is given by

$$
\frac{P_{L}}{P_{s}}=\left|\boldsymbol{S}_{21}\right|^{2}=4\left|\boldsymbol{H}_{R}\right|^{2}\left|\boldsymbol{H}_{C H}\right|^{2}\left|\boldsymbol{H}_{T}\right|^{2},
$$

in which the propagation channel can be complex e.g. due to various paths along which the signal can travel.

A commercial, off the shelf, UWB test bed [19] was used to validate the theory. The test bed includes a UWB monopole antenna (see Fig. 3, [20]) designed to operate in the frequency range $3.1 \mathrm{GHz}-$ $5.3 \mathrm{GHz}$. However, no accurate physical description of the monopole geometry and materials was available. To use this antenna in simulations, one has to build a model by measuring the physical dimensions of the antenna and by estimating the dielectric properties of the antenna substrate. In the end, this may result in a decent approximation. On the other hand, this is not really necessary. The proposed framework allows to incorporate a measured far-field vector, gain or other antenna figure of merit into the block model instead of approximate simulations. In the light of validating our theory, we apply both approaches.

To obtain the antenna transfer function, the suggested three-antenna measurement was performed with the UWB monopole [20], an UWB horn [21] and two standard gain horn ${ }^{\ddagger}$ (Scientific Atlanta's 12-2.6 and 12-3.9 standard gain horns [22]). The antenna transfer function is then calculated following the procedure in 23 . The channel transfer function in the anechoic chamber equals

$$
\boldsymbol{H}_{C H}=\boldsymbol{H}_{F S}=\frac{-2 \jmath f Z_{L}}{\eta c} \frac{\mathrm{e}^{-\jmath k d}}{d} .
$$

To validate our theory, we would like to simulate the antenna links we measured in the anechoic chamber with the time domain solver of CST-MWS ${ }^{\circledR}$. Unfortunately, this is impossible due to the large antenna spacing $(5 \mathrm{~m}$ or approximately $51 \lambda$ at $3.1 \mathrm{GHz}$ ) and the size of the horn antennas. It was, however, possible to simulate a full monopole to monopole link over a distance of $0.5 \mathrm{~m}$ $(\approx 5 \lambda)$ A distance of $0.5 \mathrm{~m}$ does not really correspond to the far-field at the considered frequencies. To verify the accuracy of the link simulation at that distance, another CST-MWS ${ }^{\circledR}$ simulation was performed at $0.75 \mathrm{~m}$, applying a less dense mesh. Then, the radiation vector resulting from the electric field observed at $0.75 \mathrm{~m}$ was compared to the one calculated at $0.5 \mathrm{~m}$. A reasonably good agreement was found between both results, verifying the far-field approximation for a distance of $0.5 \mathrm{~m}$, within a reasonable accuracy, with CST-MWS ${ }^{\circledR}$. To reproduce this result with measurement

\footnotetext{
$\ddagger_{\text {together they completely cover the required frequency range }}$
} 


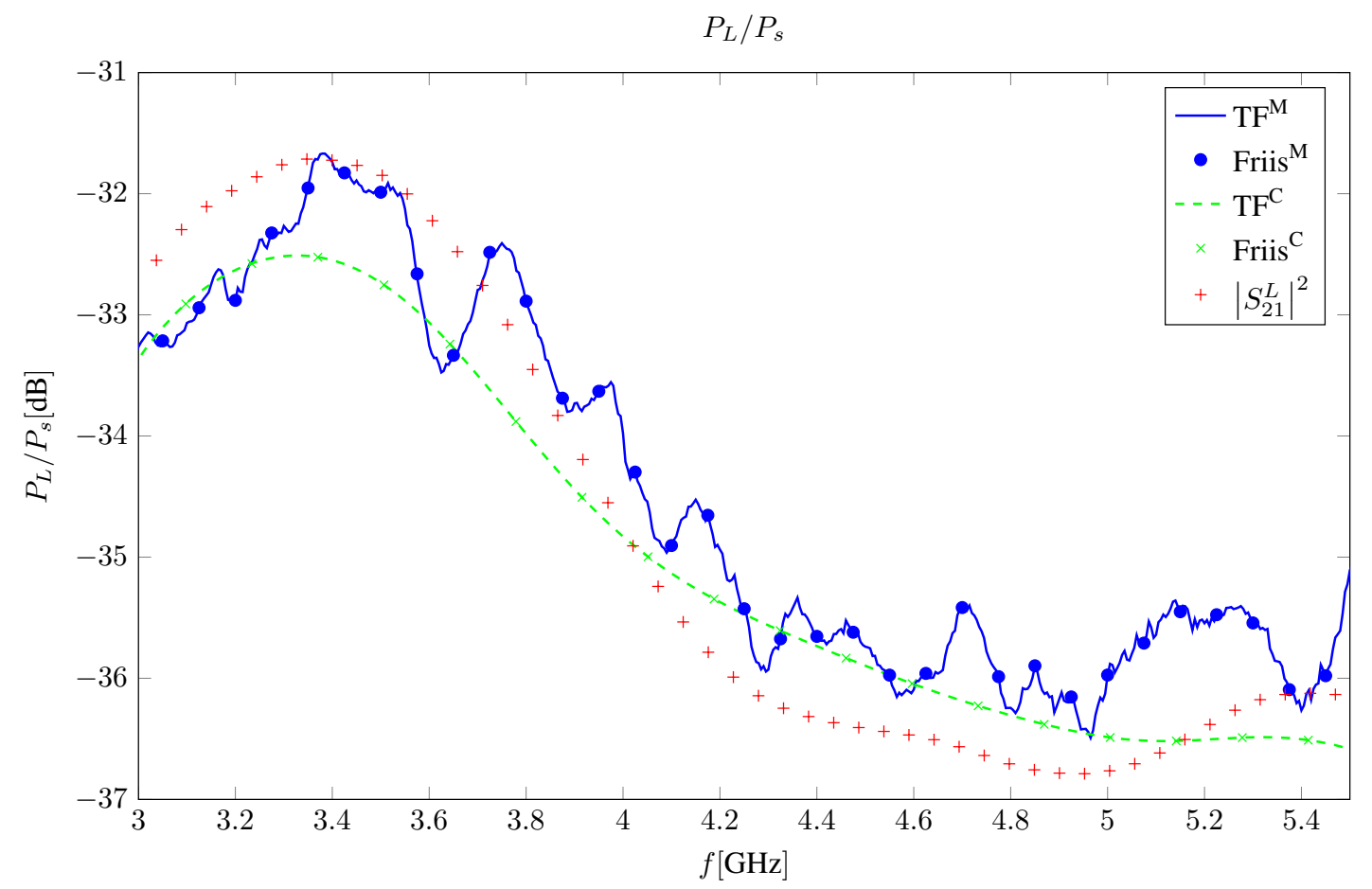

Figure 4. The power ratio $\frac{P_{L}}{P_{s}}$, calculated in different ways: using the monopole's measured transfer function $\left(\mathrm{TF}^{M}\right.$, blue, solid), using the Friis transmission formula based on measurements (Friis ${ }^{M}$, blue, dots), using the monopole's simulated (stand-alone) transfer function $\left(\mathrm{TF}^{C}\right.$, green, dashed), using the Friis transmission formula based on a stand-alone simulation (Friis ${ }^{C}$, green, crosses) and using $\left|\boldsymbol{S}_{21}\right|^{2}$ as directly simulated $\left(\left|\boldsymbol{S}_{21}^{L}\right|^{2}\right.$, red, plusses $)$

data, the monopole transfer function was extracted from the measurements and the propagation transfer function was replaced by 26 for an antenna spacing of $5 \mathrm{~m}(d=5 \mathrm{~m})$. In addition, the antenna transfer function of the monopole was also calculated from a stand-alone simulation of the antenna using (17). As an extra way of validating the theory, the Friis transmission formula was also used to calculate the power transmitted from the one monopole to the other.

Next, all the expressions for the ratio between received and transmitted power are calculated and plotted in Fig. 4. First, the power ratio was calculated using the measured monopole's transfer function $\left(\mathrm{TF}^{M}\right.$, blue, solid)

$$
\frac{P_{L}}{P_{s}}=4\left|\boldsymbol{H}^{M}\right|^{2}\left|\boldsymbol{H}_{C H}\right|^{2}\left|\boldsymbol{H}^{M}\right|^{2}
$$

and using Friis's formula with measured gains and reflection coefficients (Friis ${ }^{M}$, blue, dots)

$$
\frac{P_{L}}{P_{s}}=G^{M} \cdot M^{M} \cdot \mathrm{PL}^{-1} \cdot M^{M} \cdot G^{M}
$$

Second, the power ratio was evaluated using stand-alone simulations of the monopole antenna based on transfer functions, $\left(\mathrm{TF}^{C}\right.$, green, dashed)

$$
\frac{P_{L}}{P_{s}}=4\left|\boldsymbol{H}^{C}\right|^{2}\left|\boldsymbol{H}_{C H}\right|^{2}\left|\boldsymbol{H}^{C}\right|^{2}
$$

and based on Friis's formula (Friis ${ }^{C}$, green, crosses)

$$
\frac{P_{L}}{P_{s}}=G^{C} \cdot M^{C} \cdot \mathrm{PL}^{-1} \cdot M^{C} \cdot G^{C} .
$$




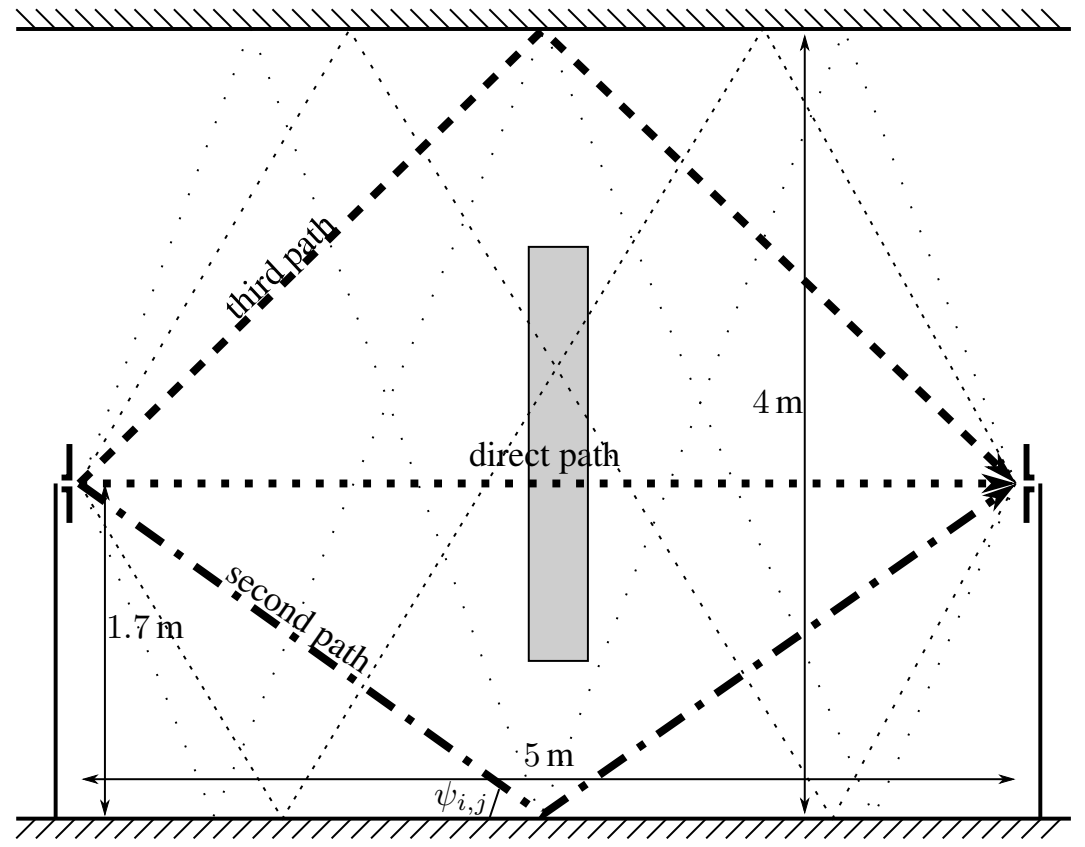

Figure 5. Simulation set-up consisting of a receiver and a transmitter $5 \mathrm{~m}$ apart both placed at a height of $1.7 \mathrm{~m}$ above ground. The direct path is represented by a dotted line, the first additional path by dash-dotted line and the third propagation path by a dashed line. The third propagation path is reflected from a $4 \mathrm{~m}$ high ceiling. The floor-ceiling combination creates a multipath environment. Four additional paths are drawn in fine dashes and fine dots. In the centre, a blocking element was placed. This will block all paths going through the centre in the non-line-of-sight case discussed later on.

Finally, the power ratio was calculated using the $S_{21}$ as simulated, without the transfer function extraction $\left(\left|S_{21}^{L}\right|^{2}\right.$, red, plusses)

$$
\frac{P_{L}}{P_{s}}=\left|\boldsymbol{S}_{21}^{L}\right|^{2}
$$

All antenna figures of merit used above relate to the UWB monopole and PL represents the path loss. The full link simulation in CST-MWS ${ }^{\circledR}$ lasted $4 \mathrm{~h} 4 \mathrm{~m} 14 \mathrm{~s}$ on a quad core system and used over 6.5 GB of RAM memory. A stand-alone simulation of the antenna only lasted $39 \mathrm{~m} 25 \mathrm{~s}$ on the same system and used 3.7 GB RAM memory. The combination of the correct transfer functions took only seconds.

One can see that all data are in good agreement. Differences between simulated data are due to the different meshes CST-MWS ${ }^{\circledR}$ applied to different set-ups and to the fact that the antenna separation does not really satisfy the far-field condition. Allowing for reflections on different surfaces complicates the basic configuration above. Adding a concrete floor $1.7 \mathrm{~m}$ below both transmitter and receiver results in one additional propagation path next to the direct path. This second path is indicated as such in Fig. 5 This set-up will be called the 'two-path' configuration. Adding a concrete ceiling $4 \mathrm{~m}$ above the floor gives raise to a, theoretically, infinite number of additional propagation paths between transmitter and receiver. The first three paths are depicted and labelled in Fig. 5. This set-up will be named 'three-path' configuration. The other paths, of which a few are also depicted, reflect twice, thrice and so on, on floor and ceiling. With every additional reflection, the propagation path becomes longer and its contribution to the power at the receiver diminishes. As seen in Fig. 6, extensive simulations show that, compared to a scenario that includes all reflected rays ( 50 are considered in practice $\$$, the relative error on the ratio between received and transmitted power is of the order of $10^{-10}$ when only fifteen propagation paths are considered. In

\footnotetext{
$\S$ considering more than 50 paths did not change the result within the computer's accuracy
} 


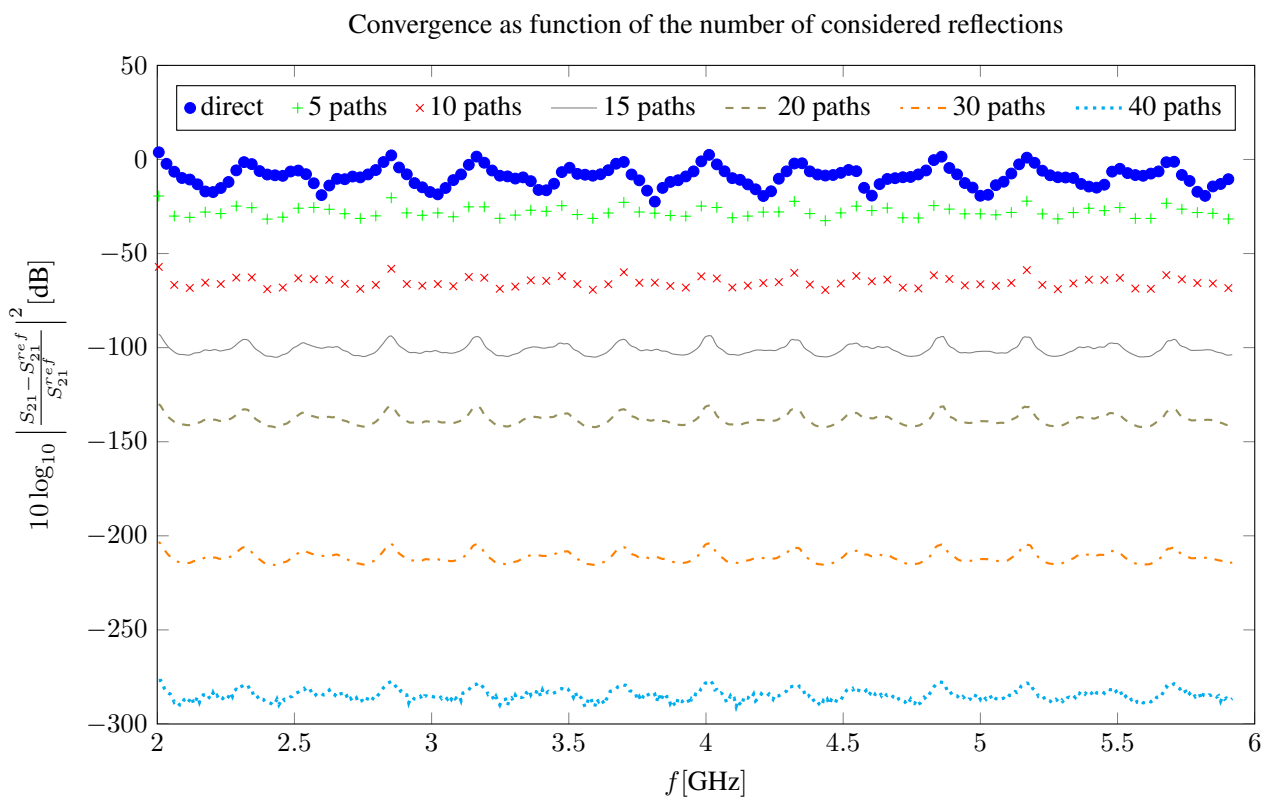

Figure 6. Convergence of the result as a function of the number of considered reflections. The used measure is the relative error with respect to a reference solution. As a reference solution, 50 reflections were taken into account, adding more reflections did not change the result within the computer accuracy.

addition, a blocking element can be added in the the midpoint between the two antennas, changing the proposed set-up from a LOS to a NLOS configuration. The blocking element, depicted as a light grey box in Fig. 5, will block all propagation paths going through the middle, clearly obstructing the direct path and all paths with an even number of reflections. In Fig. 7, the ratio of the received power over the transmitted power versus the frequency is depicted. One can clearly observe that for the two-path configuration, the power ratio fluctuates around the result of the direct path. Peaks and valleys are spaced approximately $141 \mathrm{MHz}$ apart, corresponding with a wavelength of $1.06 \mathrm{~m}$, which almost equals the the path length difference $(\Delta d)$ between the direct path and the reflected path of $1.05 \mathrm{~m}$. For the three-path configuration, one cannot easily predict the result based on simple $\Delta d$ data. In this case the presented tool shows how the power ratio fluctuates around the direct path result. One can clearly see that the peaks and valleys are more distinct and unpredictable, compared to the two-path configuration. The NLOS case, as depicted in Fig. 8, has no direct path, since it is blocked by the element in the centre of the simulation space (see Fig. 5). The two-path configuration cannot interfere with the non-existent direct path, and therefore the power ratio looks like the direct path result in Fig. 7. However, about $10 \mathrm{~dB}$ less power is received due to the longer path length and the power loss caused by the reflection. Many propagation paths interfere with one another in the three-path configuration. The resulting power ratio shows clear peaks and valleys, separated by approximately $400 \mathrm{MHz}$, corresponding to a wavelength of $0.74 \mathrm{~m}$. This is about the path length difference, $\Delta d=0.75 \mathrm{~m}$, between the two major contributions to the result, being the path that reflects once on the floor and the path that reflects once on the ceiling.

\section{APPLICATION TO BODY-CENTRIC WPT}

In the previous section, it was shown above that the proposed framework yields accurate results. We now apply it to determine the ideal distance between transmitter and receiver to optimize the power transmission. Let us consider the multipath configurations introduced above, taking into account said multipath environment and additional shadowing by the human body. To do this, we place the receive antenna on the chest of a test person $(1.75 \mathrm{~m}, 70 \mathrm{~kg})$. The antenna was taped to the shirt 


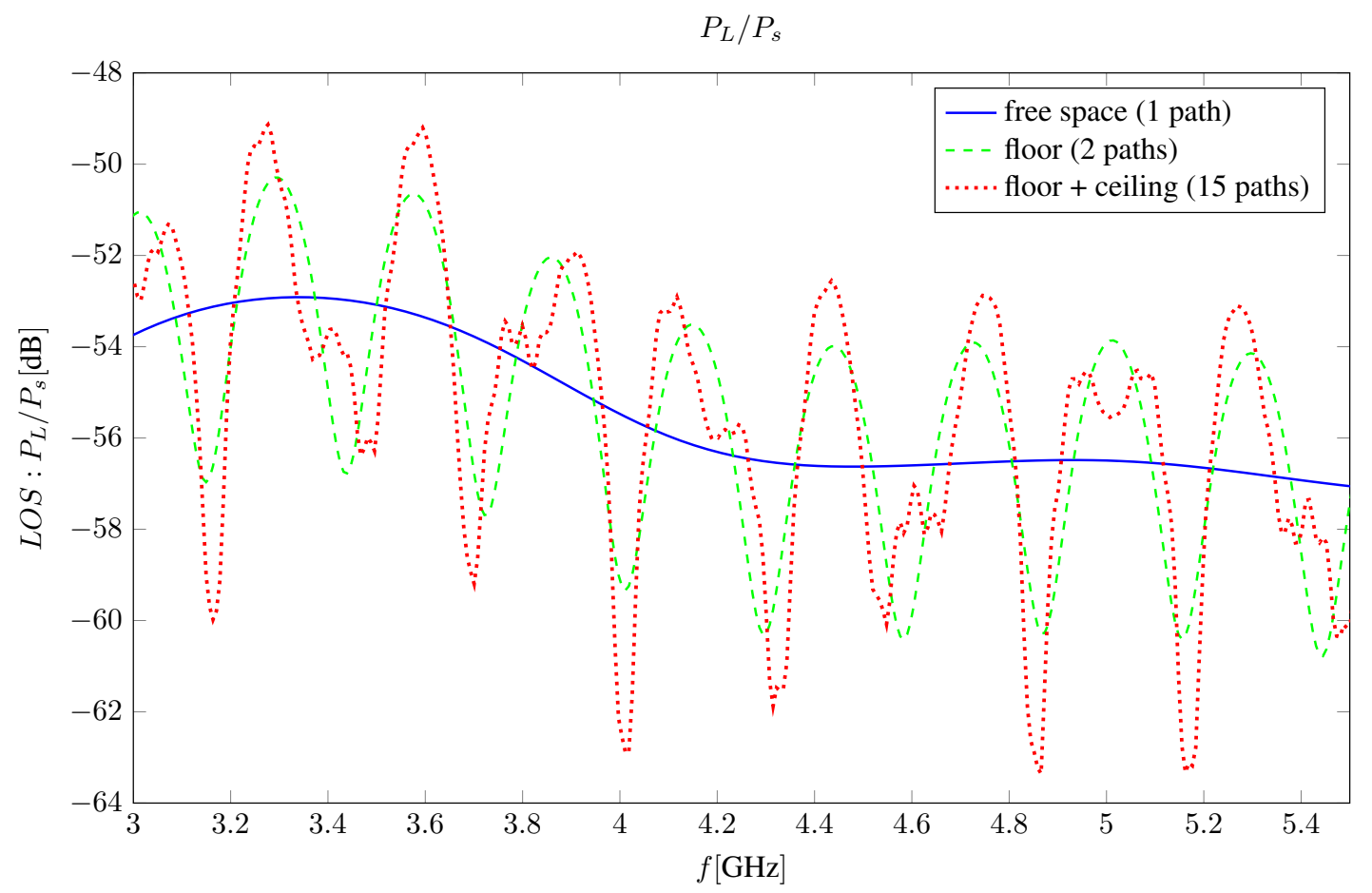

Figure 7. Power transfer over three different LOS channels with a length of $5 \mathrm{~m}$ : free space propagation (Blue, solid), two-path model, sender and receiver are placed $1.7 \mathrm{~m}$ above ground (green, dashed) and a model consisting of the two-path model extended with a ceiling $4 \mathrm{~m}$ above ground (red, dotted).

of the test person and spacer fabrics were used to increase the spacing between body and antenna. Remark that when no spacer fabric was used, the antenna was deployed 2 to $3 \mathrm{~mm}$ (estimate) away from the body, because the antenna was positioned on a loose shirt. The linear UWB monopole was placed on the body in such a way that its polarisation was vertical and aligned with the vertical polarisation of the UWB horn [21] used as transmit antenna in this measurement. Therefore, the horizontal polarised component in the measured $\boldsymbol{S}_{21}$ is very small and is neglected from here on. On-body data will be indicated by a subscript $b$.

First of all, the system was excited with an UWB pulse, depicted in Fig. 9, with a duration of $6.1 \mathrm{~ns}$. The pulse is normalized to have a power of $1 \mathrm{~W} .99 .95 \%$ of the power of the pulse is concentrated in a time frame between $0.47 \mathrm{~ns}$ and $4.34 \mathrm{~ns}$, as indicated by red dashes in Fig. 9

Second, the transfer function of the monopole antenna on the human body was measured $\left(\boldsymbol{H}_{b}^{M}\right.$, see Fig. 10) for distances varying between $3 \mathrm{~mm}$ and $20 \mathrm{~mm}$. Third, the transfer function was also simulated with CST-MWS ${ }^{\circledR}\left(\boldsymbol{H}_{b}^{C}\right.$, see Fig. 10). In the simulations, the human body was represented by a three layer ((dry) skin $(1 \mathrm{~mm})$, fat $(3 \mathrm{~mm})$ and muscle $(18 \mathrm{~mm}))$ tissue model of size $150 \times 150 \mathrm{~mm}$. The frequency dependent dielectric properties of the body tissues were obtained from [23].

In Fig. 10, a selection of the measured and simulated transfer functions of the monopole on the human body is depicted. One can see that, except for the simulation at $3 \mathrm{~mm}$ separation, the body acts as a reflector. Therefore, the received power near the human body is higher than in free space. Note that for the measurement at $10 \mathrm{~mm}$ distance from the human body, the transfer function starts to show a dip, but the deepest point lies outside the measurement range. This is not seen in the simulations. Other simulations have shown that the dip occurs at higher frequencies, well outside the frequency band of interest. The discrepancies between measurements and simulations are probably caused by the use of the three layer model in CST-MWS ${ }^{\circledR}$.

Let it be clear that it is extremely difficult to accurately model the interaction of electromagnetic radiation with the human body. Besides, the simulations of the antenna on the human body 
NLOS: $P_{L} / P_{s}$

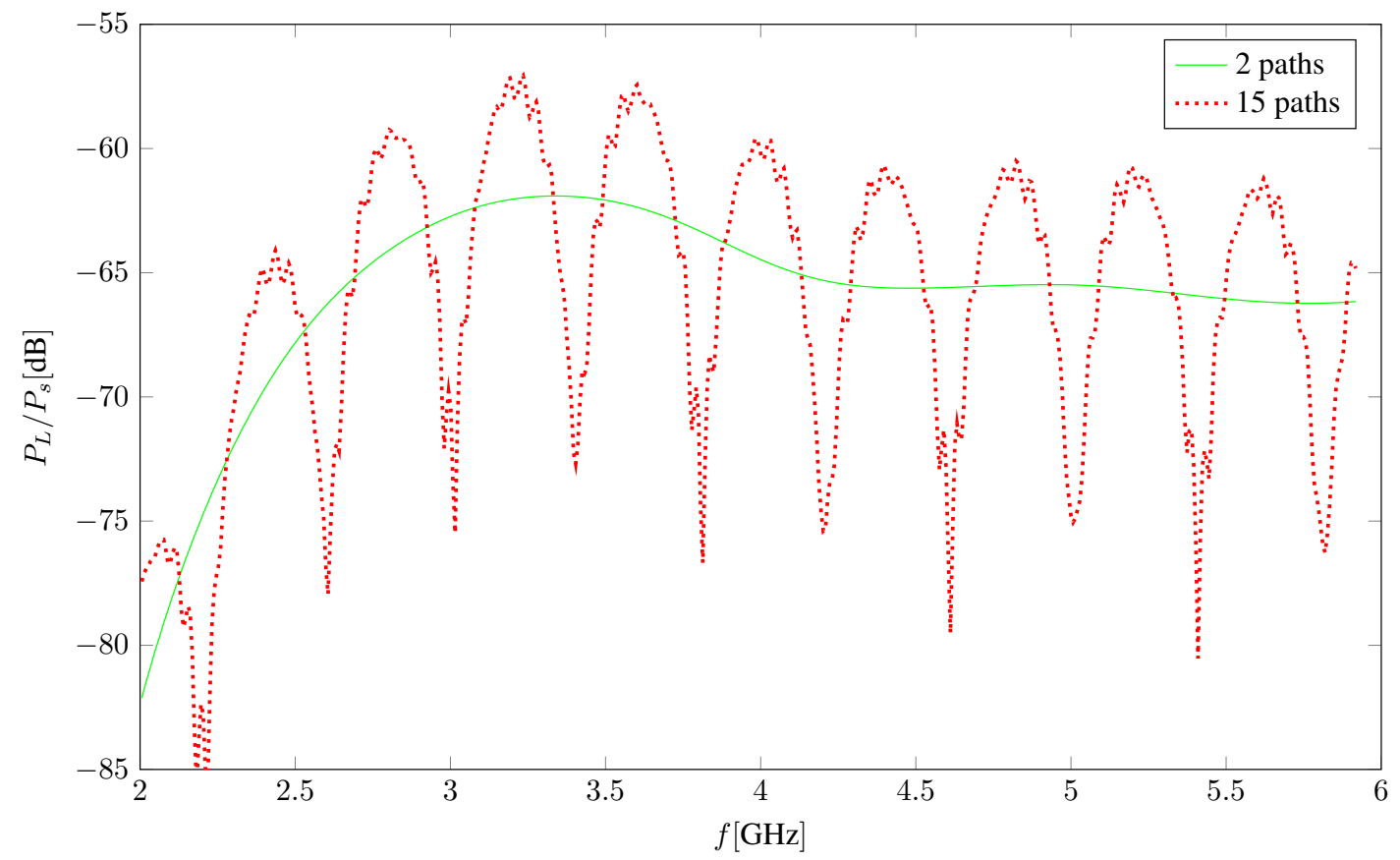

Figure 8. Power transfer over two different channels with a length of $5 \mathrm{~m}$ : two-path model, sender and receiver are placed $1.7 \mathrm{~m}$ above ground (green, dashed) and a model consisting of the two-path model extended with a ceiling $4 \mathrm{~m}$ above ground (red, dotted). All paths going through the middle are blocked as depicted in Fig. 5

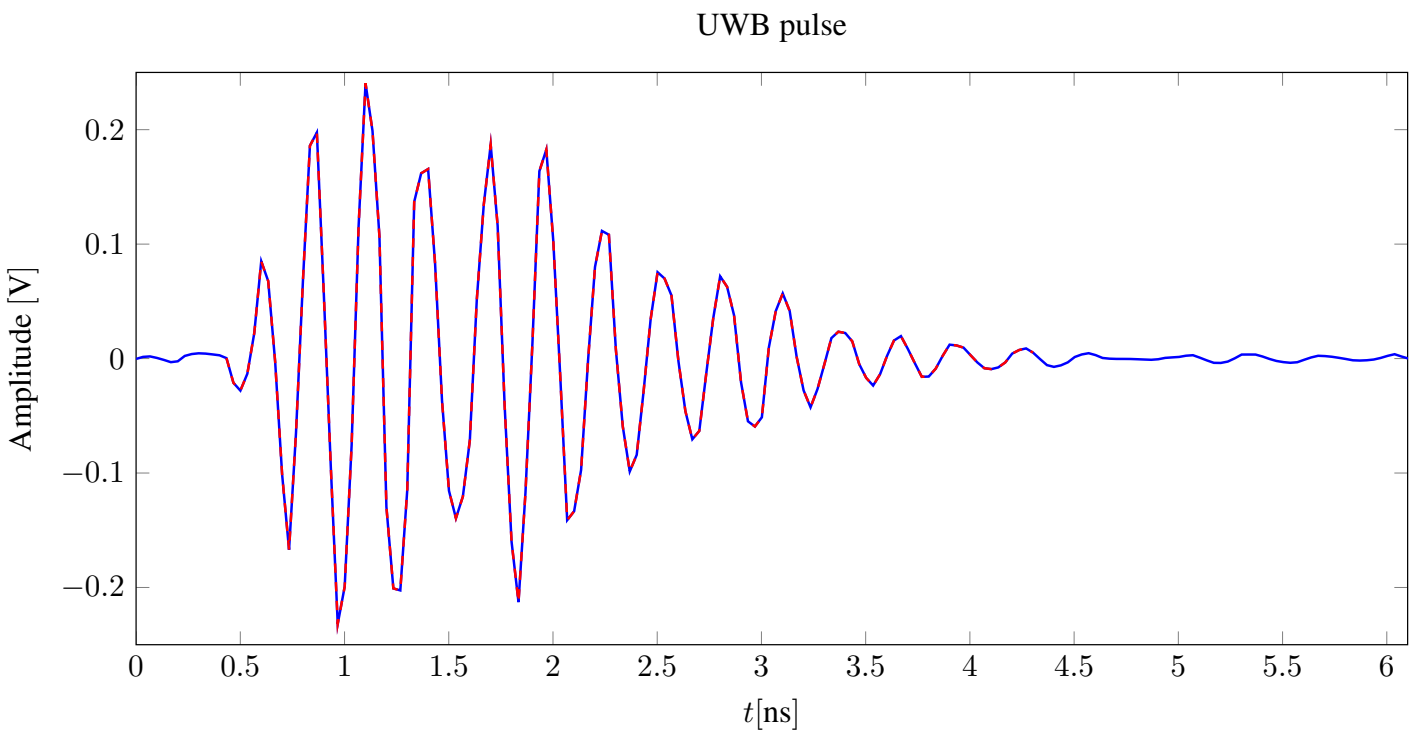

Figure 9. UWB pulse (blue). The part containing $99.95 \%$ of pulse's power is marked with red dashes (between $0.47 \mathrm{~ns}$ and $4.34 \mathrm{~ns}$ ).

were also time and memory consuming. Fortunately, the tedious and to a greater or lesser extent inaccurate process of running the on-body simulations, can be entirely circumvented by using the proposed framework and by plugging in measurements of the antenna deployed on the body into the simulation. Using the on-body measurements, the power delivered to the on-body antenna $\left(P_{L}\right)$ 


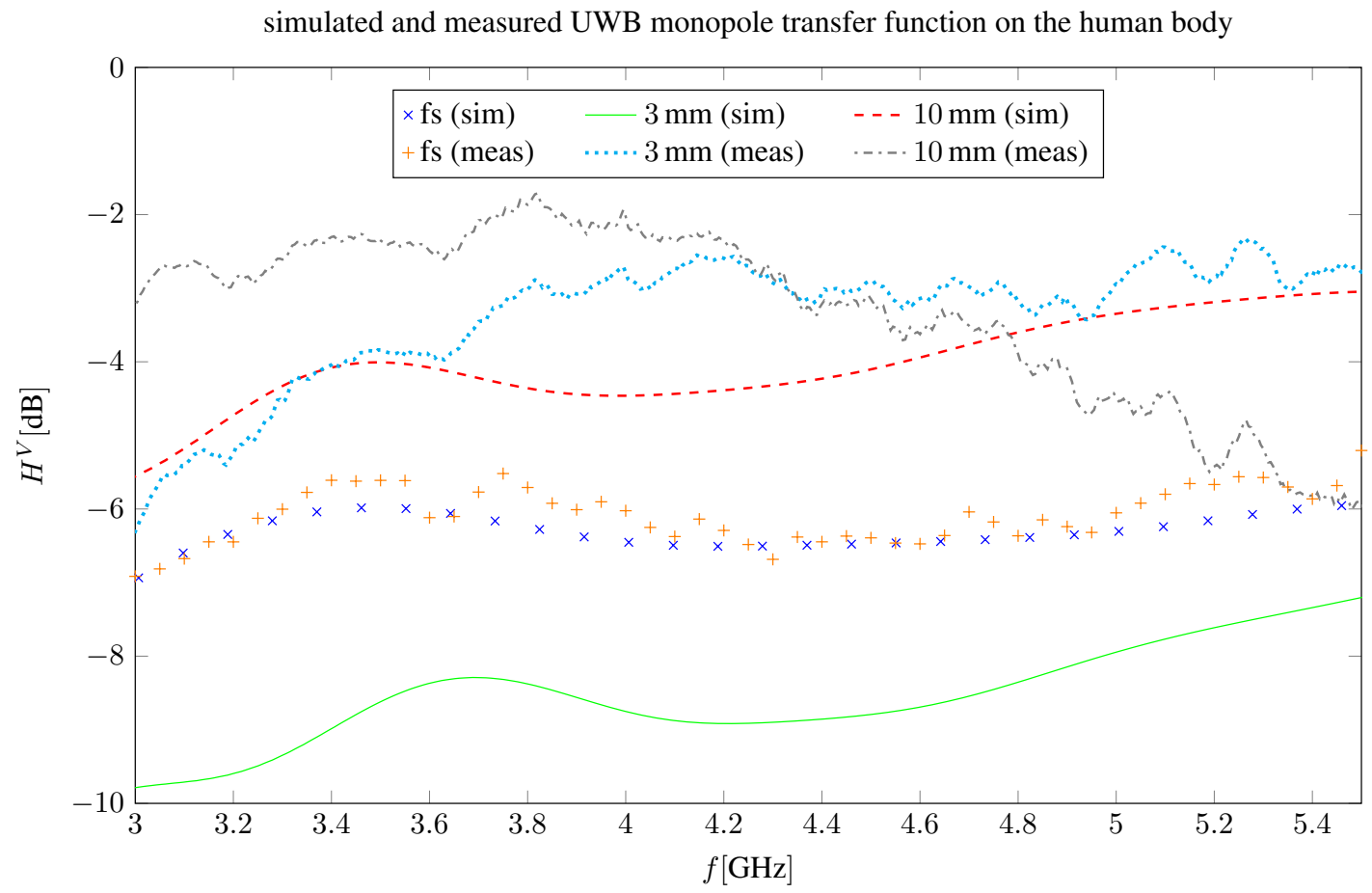

Figure 10. The transfer function of the UWB monopole placed on the human body from both simulations and measurements at distances of $3 \mathrm{~mm}$ and $10 \mathrm{~mm}$. As a reference the transfer function of the free-standing monopole (simulated: blue crosses and measured: orange plusses) is added.

by the free-standing antenna $\left(P_{s}\right)$ is expressed by (with $\boldsymbol{H}^{C}$ the stand-alone simulated monopole transfer function in free space)

$$
P_{L}=4 \cdot\left|\boldsymbol{H}_{b}^{M}\right|^{2} \cdot\left|\boldsymbol{H}_{C H}\right|^{2} \cdot\left|\boldsymbol{H}^{C}\right|^{2} P_{s} .
$$

Finally, to put emphasis on the difference between a single direct propagation path and two-path propagation, the results are normalized with respect to the (direct) distance $(d)$ between transmitter and receiver. This means that the result is divided by the energy transmitted along the direct path. Therefore, if the result is larger (smaller) than one, more (less) energy is transported by the two-path system compared to the direct propagation scenario. The differences between multipath and direct path propagation are caused by the $\Delta d$ (rather than by the direct propagation distance $d$ ). Therefore results will be presented as a function of $\Delta d$ (rather than $d$ ), as compiled in Table $\mathbb{I}$.

In Fig. 11, the received power is plotted as a function of $\Delta d$ for a two-path link. First note that the effect of the second path is almost independent of the spacing between the antenna and the human body. One can observe that, for $\Delta d>1.3 \mathrm{~m}$, the curve is flat and slightly larger than one. This means that the second path delivers additional power to the receive antenna (compared to only a direct path). A $\Delta d$ of $1.3 \mathrm{~m}$ corresponds to a time delay between the two pulses of $4.34 \mathrm{~ns}$. Since almost all the energy of the pulse is concentrated in a smaller time frame, no interference occurs. A $\Delta d$ of $1.3 \mathrm{~m}$ corresponds to a direct path length of $3.79 \mathrm{~m}$ (given that transmitter and receiver are placed at a height of $1.7 \mathrm{~m}$ )

When $\Delta d$ drops below $1.3 \mathrm{~m}$, the pulses received over both propagation paths overlap more and more and a pattern of constructive and destructive interference appears. The pattern is fairly regular, the constructive interference peaks increase with decreasing $\Delta d$, while the destructive interference dips deepen. The distance between peaks is fixed at about $0.085 \mathrm{~m}$. The highest additional relative power transmission caused by the second path occurs for a $\Delta d$ of $0.042 \mathrm{~m}$.

Similar to the adopted approach at the end of Section 3 a concrete ceiling is added $4 \mathrm{~m}$ above the floor. The results for the received power in the LOS case are displayed in Fig. 12. Note that $\Delta d$ is not 


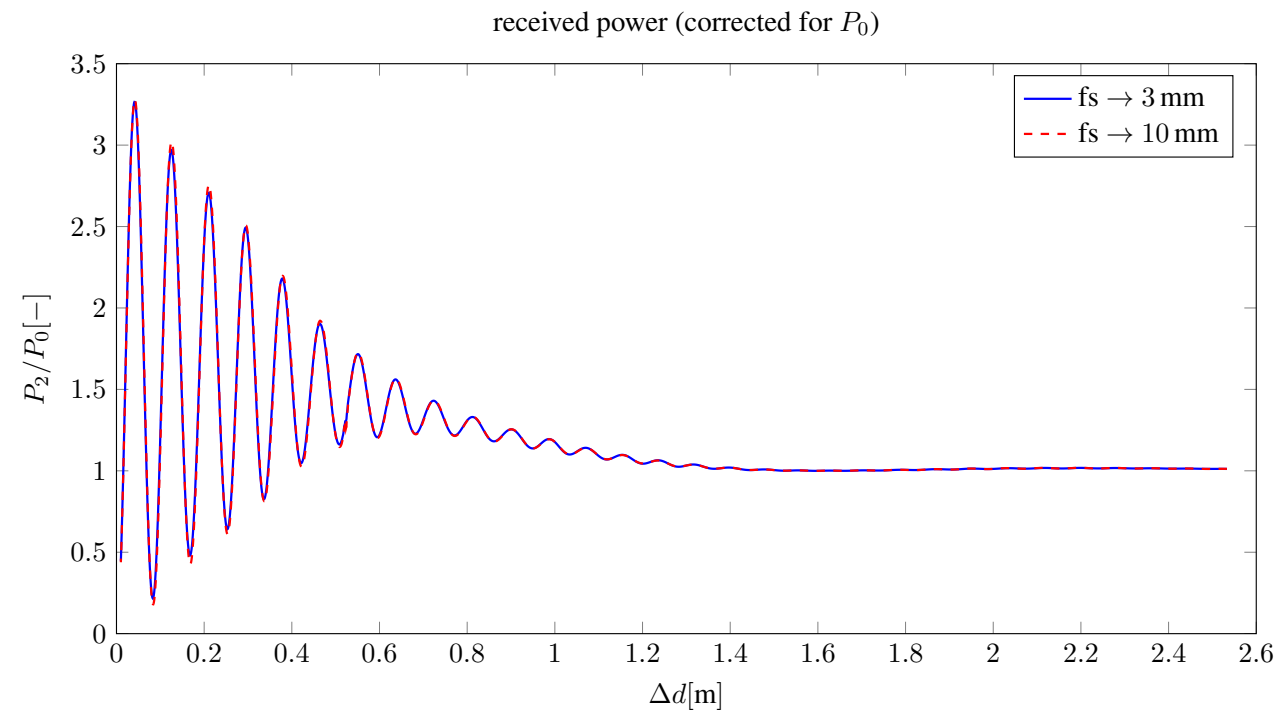

Figure 11. Received power versus $\Delta d$ for the two-path configuration $\left(P_{2}, \mathrm{Tx} \rightarrow \mathrm{Rx}\right.$, free-standing (fs) to on-body), corrected for the power received via the direct path $\left(P_{0}\right)$.

\begin{tabular}{rc}
\hline configuration & optimal $\Delta d[\mathrm{~cm}]$ \\
\hline on-body 2-ray & 4.2 \\
on-body 3-ray LOS & 2.7 \\
on-body 3-ray NLOS & 10.3 \\
\hline
\end{tabular}

Table I. Table listing the optimal position to receive maximal power relative to a free space channel for a receiver placed $3 \mathrm{~mm}$ and $10 \mathrm{~mm}$ away from the human body, respectively.

uniquely defined, because there is a difference between the direct path and all the indirect paths. We chose $\Delta d$ to be the difference between the direct path and the first reflected path (as above). Adding thirteen propagation paths on top of the two in the two-path configuration, destroys the regularity observed in the two-path case. Now it is impossible to easily predict the next peak in the received power with a simple formula. But the proposed framework solves this problem. From Fig. 12 one can see that the highest additional relative power transmission via the multiple paths occurs for a $\Delta d$ of $0.027 \mathrm{~m}$. In the case of the NLOS channel, the situation differs. Now most power is received if $\Delta d$ equals $10.3 \mathrm{~cm}$ as can be seen in Fig. 13

\section{CONCLUSION AND FUTURE RESEARCH}

In this paper, a framework for the simulation of UWB, body-centric WPT in multipath configurations was presented. It was shown that the framework provides accurate results for the simulations of antenna links and power transmitted over them in multipath environments. As a means of validating the theory, a free space link was first investigated and it was found that results of measurements and simulations were in good agreement. Moreover, it was observed that, compared to the link full 3D simulation, the stand alone antenna simulations took only $17 \%$ of the time, consuming only $57 \%$ of the memory. The framework calculations then only took seconds. Next, the presented framework was applied to model the wirelessly transferred power to a wearable antenna deployed on the human body in in LOS and NLOS set-ups. Two numerical examples were presented. The first, handled body-centric WPT with a second propagation path. The results showed that for a path length difference of $4.2 \mathrm{~cm}$ the most power was received. This example was expanded to a 


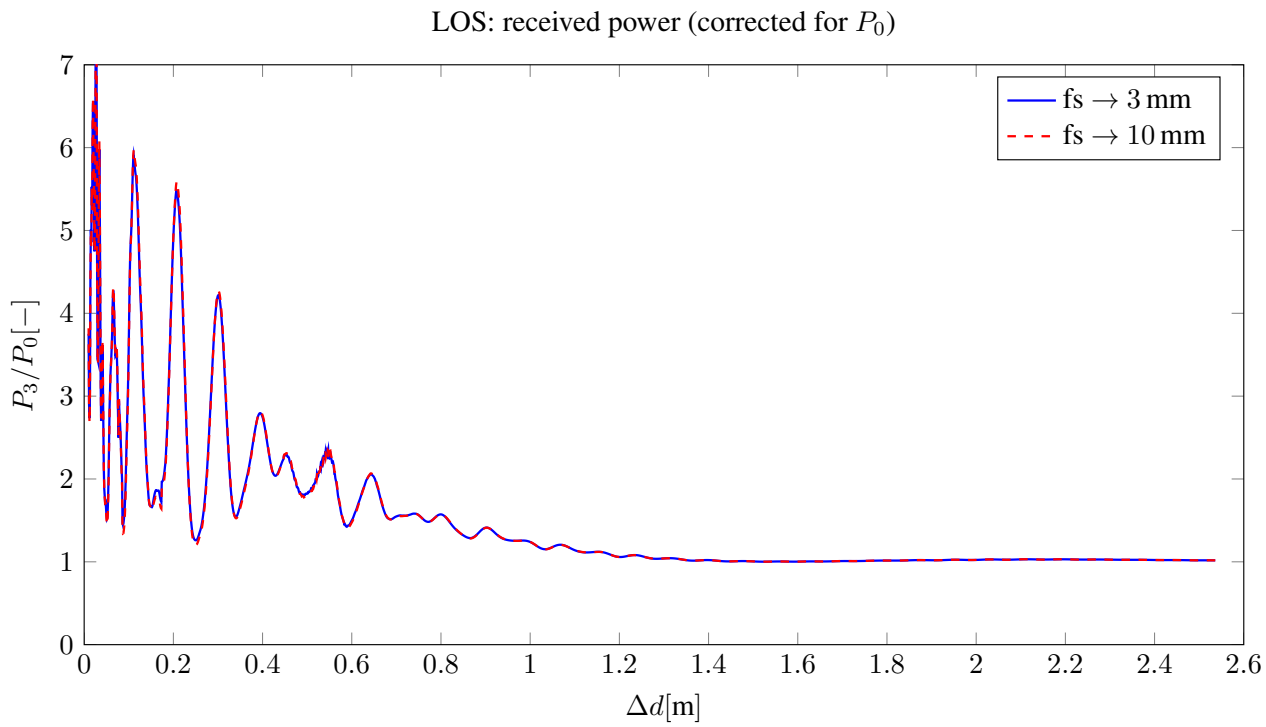

Figure 12. Received power versus $\Delta d$ for the three-path configuration $\left(P_{3}, \mathrm{Tx} \rightarrow \mathrm{Rx}\right.$, free-standing (fs) to on-body), corrected for the power received via the direct path $\left(P_{0}\right)$, in the LOS case.

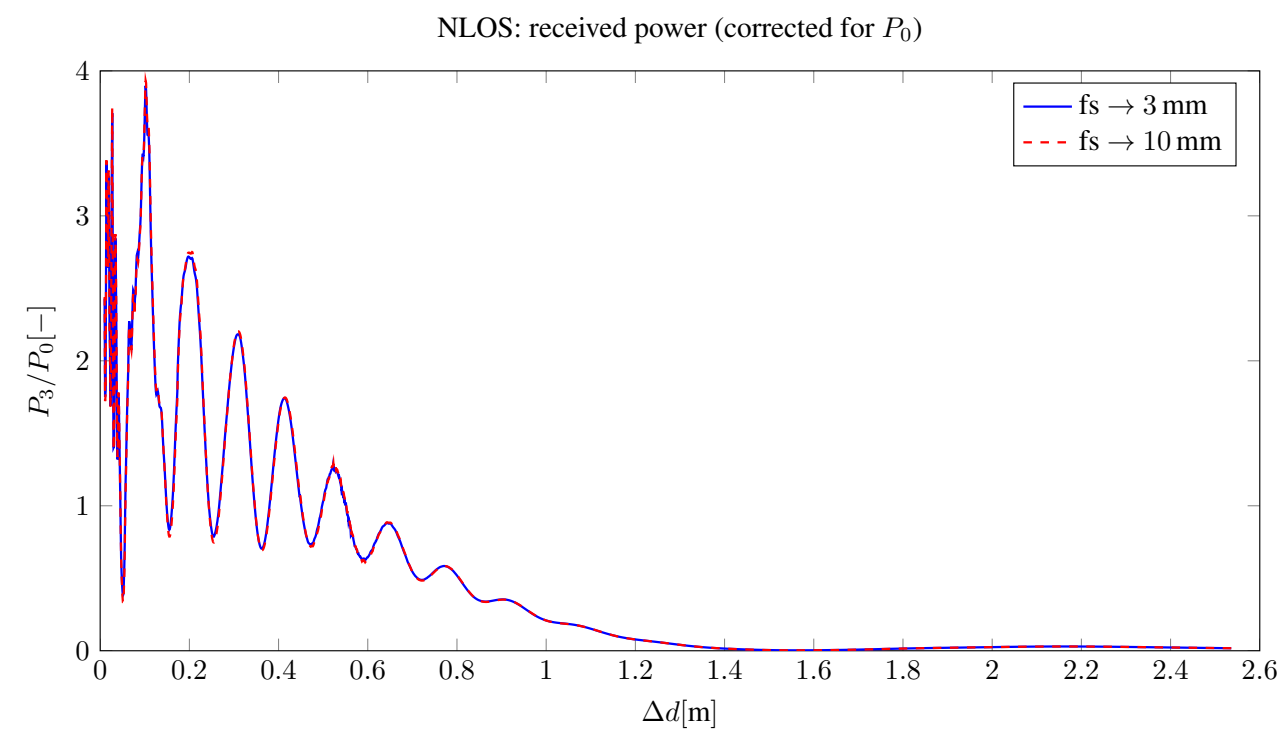

Figure 13. Received power versus $\Delta d$ for the three-path configuration $\left(P_{3}, \mathrm{Tx} \rightarrow \mathrm{Rx}\right.$, free-standing (fs) to on-body), corrected for the power received via the direct path $\left(P_{0}\right)$, in the NLOS case.

channel with fifteen paths. Then it was found that the largest amount of power was received if the path length difference equalled $2.7 \mathrm{~cm}$ in the LOS case and $10.3 \mathrm{~cm}$ in the NLOS case.

Continued research should include more channel effects such as polarisation cross-over and diffraction, in order to model and investigate more complex propagation channels. This should then allow for the detailed study of e.g. multipath cancellation. Furthermore, the power finally delivered to the power harvester (as depicted in Fig. 1) should be investigated by also including the intermediate stages such as the matching and rectifier circuitry. 
Funds for this research were obtained from the Agency for Innovation by Science and Technology in Flanders (IWT).

\section{References}

1. De Vita G, Iannaccone G. Design criteria for the RF section of UHF and microwave passive RFID transponders. Microwave Theory and Techniques, IEEE Transactions on Sept 2005; 53(9):2978-2990, doi:10.1109/TMTT.2005. 854229.

2. Dolgov A, Zane R, Popovic Z. Power Management System for Online Low Power RF Energy Harvesting Optimization. Circuits and Systems I: Regular Papers, IEEE Transactions on July 2010; 57(7):1802-1811, doi: 10.1109/TCSI.2009.2034891.

3. Brown WC. The History of Power Transmission by Radio Waves. Microwave Theory and Techniques, IEEE Transactions on Sep 1984; 32(9):1230-1242, doi:10.1109/TMTT.1984.1132833.

4. Walsh C, Rondineau S, Jankovic M, Zhao G. A Conformal $10 \mathrm{GHz}$ Rectenna for Wireless Powering of Piezoelectric Sensor Electronics 2005. URL http://ecee.colorado.edu/microwave/docs/publications/ 2005/2005RectennaPaper.pdf

5. Wireless Power Consortium. Qi standard 2009. URLhttp://www.wirelesspowerconsortium. com/

6. Power Matters Alliance. PMA Inductive Wireless Power and Charging Specifications for Receiver (Rx), Transmitter (Tx), and Host Control Interface (HCI). URL http: / / www . powermatters . org/

7. Rizzoli V, Costanzo A, Masotti D, Spadoni P, Neri A. Prediction of the End-to-End Performance of a Microwave/RF Link by Means of Nonlinear/Electromagnetic Co-Simulation. Microwave Theory and Techniques, IEEE Transactions on Dec 2006; 54(12):4149-4160, doi:10.1109/TMTT.2006.885566.

8. Ahmed N, Kanhere S, Jha S. Utilizing Link Characterization for Improving the Performance of Aerial Wireless Sensor Networks. Selected Areas in Communications, IEEE Journal on August 2013; 31(8):1639-1649, doi: 10.1109/JSAC.2013.130825.

9. Sani A, Alomainy A, Hao Y. Characterisation of ultra wideband wearable antennas and body-centric wirelessnetworks in indoor environment. Wireless Technology, 2008. EuWiT 2008. European Conference on, Amsterdam, The Netherlands, 2008; 135-138.

10. Di Bari R, Abbasi QH, Alomainy A, Hao Y. An Advanced UWB Channel Moel For Body-Centric Wireless Networks. Progress In Electromagnetics research 2013; 136:79-99.

11. Miao Y, Haneda K, Kim M, Takada JI. Stochastic modelling of LOS double-directional channel in spherical wave domain - Concept, formulation and validation. General Assembly and Scientific Symposium (URSI GASS), 2014 XXXIth URSI, China National Convention Center, No.7 Tianchen East Road, Chaoyang District, Beijing, China, 2014; 1-1, doi:10.1109/URSIGASS.2014.6929656.

12. Alomainy A, Hao Y, Owadally A, Parini C, Nechayev Y, Constantinou C, Hall P. Statistical Analysis and Performance Evaluation for On-Body Radio Propagation With Microstrip Patch Antennas. Antennas and Propagation, IEEE Transactions on Jan 2007; 55(1):245-248, doi:10.1109/TAP.2006.888462.

13. Hall PS, Hao Y. Antennas and Propagation for Body-Centric Wireless Communications. 2 edn., Artech House: Norwood, MA 02062 USA, 2012.

14. Bahrami H, Gosselin B, Rusch L. Realistic modeling of the biological channel for the design of implantable wireless UWB communication systems. Engineering in Medicine and Biology Society (EMBC), 2012 Annual International Conference of the IEEE, Osaka, Japan, 2012; 6015-6018, doi:10.1109/EMBC.2012.6347365.

15. Wiesbeck W, Adamiuk G, Sturm C. Basic Properties and Design Principles of UWB Antennas. Proceedings of the IEEE Feb 2009; 97(2):372-385, doi:10.1109/JPROC.2008.2008838.

16. Promwong S, Panthap P. Experimental evaluation of complex form Friis' transmission formula with indoor/outdoor for ultra wideband inpulse radio. Computer and Communication Engineering, 2008. ICCCE 2008. International Conference on, International Islamic University Malaysia, Kuala Lumpur, Malaysia, 2008; 1037-1041, doi: 10.1109/ICCCE.2008.4580766.

17. Agu E, Hill FS. A Simple Method for Ray Tracing Diffraction. Proceedings of the 2003 International Conference on Computational Science and Its Applications: PartIII, ICCSA'03, Springer-Verlag: Berlin, Heidelberg, 2003; 336-345. URL http://dl.acm.org/citation.cfm?id=1761792.1761832

18. Ait-Ighil M, Rougerie S, Lemorton J, Carrie G, Artaud G, Bourga C, Bousquet M. Channel modelling in complex urban environments for testing multipath mitigation methods enhanced by antenna array. Space Communications $2013 ; 22(2-4): 111-123$.

19. Time Domain. Datasheet UWB Ranging Radio. URL http://www.timedomain.com/datasheets/ 320-0289EP410Datasheet.pdf

20. Time Domain. Datasheet PulseOn 200. URL http://www.timedomain.com/datasheets/TD_ Broadspec_Antenna.pdf

21. Rhode \& Schwartz. Datasheet double ridged UWB horn HF906. URL http: / / cdn. rohde-schwarz . com/ pws/dl_downloads/dl_common_library/dl_brochures_and_datasheets/pdf_1/HF906 brief e.pdf

22. Scientific Atlanta. Datasheet 12-2.6 and 12-3.9 standard gain horns. URL http://www.atecorp.com/ ATECorp/media/pdfs/data-sheets/Scientific-Atlanta-Series12_Datasheet.pdf

23. Andreuccetti D, Fossi R, Petrucci C. An Internet resource for the calculation of the dielectric properties of body tissues in the frequency range $10 \mathrm{~Hz}-100 \mathrm{GHz}$ 1997. URL/http://niremf.ifac.cnr.it/tissprop/ 